\title{
Numerical Simulations of Convective Accretion Flows in Three Dimensions
}

\section{Citation}

Igumenshchev, Igor V., Marek A. Abramowicz, and Ramesh Narayan. 2000. "Numerical Simulations of Convective Accretion Flows in Three Dimensions." The Astrophysical Journal 537 (1): L27-30. https://doi.org/10.1086/312755.

\section{Permanent link}

http://nrs.harvard.edu/urn-3:HUL.InstRepos:41384927

\section{Terms of Use}

This article was downloaded from Harvard University's DASH repository, and is made available under the terms and conditions applicable to Other Posted Material, as set forth at http:// nrs.harvard.edu/urn-3:HUL.InstRepos:dash.current.terms-of-use\#LAA

\section{Share Your Story}

The Harvard community has made this article openly available.

Please share how this access benefits you. Submit a story.

Accessibility 


\title{
Numerical Simulations of Convective Accretion Flows in Three Dimensions
}

\author{
Igor V. Igumenshchev ${ }^{1,2}$, Marek A. Abramowicz ${ }^{1,3}$, and Ramesh Narayan ${ }^{4}$
}

\begin{abstract}
Advection-dominated accretion flows (ADAFs) are known to be convectively unstable for low values of the viscosity parameter $\alpha$. Two-dimensional axisymmetric hydrodynamic simulations of such flows reveal a radial density profile which is significantly flatter than the $\rho \propto r^{-3 / 2}$ expected for a canonical ADAF. The modified density profile is the result of inward transport of angular momentum by axisymmetric convective eddies. We present three-dimensional hydrodynamic simulations of convective ADAFs which are free of the assumption of axisymmetry. We find that the results are qualitatively and quantitatively similar to those obtained with two-dimensional simulations. In particular, the convective eddies are nearly axisymmetric and transport angular momentum inward.
\end{abstract}

Subject headings: accretion, accretion disks — black hole physics — convection hydrodynamics - turbulence

\section{Introduction}

Analytical and numerical calculations have shown that advection-dominated accretion flows (ADAFs) experience strong convection, especially for low values of the viscosity parameter $\alpha$ (Narayan \& Yi 1994, 1995; Igumenshchev, Chen \& Abramowicz 1996; Igumenshchev \& Abramowicz 1999, 2000; Stone, Pringle \& Begelman 1999). The numerical work reveals that convection significantly modifies the structure of ADAFs (Stone et al. 1999; Narayan, Igumenshchev \& Abramowicz 2000, hereafter NIA). The change in the structure is understood to be a consequence of inward transport of angular momentum by convective eddies (NIA; Quataert \& Gruzinov 2000, hereafter QG; Ryu \& Goodman 1992; Stone \& Balbus 1996).

\footnotetext{
${ }^{1}$ Institute for Theoretical Physics, Göteborg University and Chalmers University of Technology, 41296 Göteborg, Sweden; ivi@fy.chalmers.se, marek@fy.chalmers.se.

${ }^{2}$ Institute of Astronomy, 48 Pyatnitskaya Street, 109017 Moscow, Russia.

${ }^{3}$ Laboratorio Interdisciplinare SISSA, Trieste, Italy, and ICTP, Trieste, Italy.

${ }^{4}$ Harvard-Smithsonian Center for Astrophysics, 60 Garden Street, Cambridge MA 02138, U. S. A.; narayan@cfa.harvard.edu.
} 
All the numerical simulations done so far have corresponded to axisymmetric two-dimensional flows. NIA and QG emphasized that the inward transport of angular momentum seen in these simulations is a consequence of axisymmetry (cf. Stone \& Balbus 1996) and that the results could in principle be qualitatively very different in three dimensions. We present in this paper the first fully three-dimensional numerical simulations of ADAFs. The details of our numerical technique are given in $\S 2$ and the results are described in $\S 3$. The paper concludes with a discussion in $\S 4$.

\section{Numerical Method}

We solve the non-relativistic time-dependent Navier-Stokes equations of an accretion flow in the gravitational potential of a central black hole:

$$
\begin{gathered}
\frac{d \rho}{d t}+\rho \nabla \cdot \vec{v}=0, \\
\rho \frac{d \vec{v}}{d t}=-\nabla P+\rho \nabla \Phi+\nabla \mathbf{\Pi}, \\
\rho \frac{d e}{d t}=-P \nabla \cdot \vec{v}+Q,
\end{gathered}
$$

where $\rho$ is the density, $\vec{v}$ is the velocity, $P$ is the pressure, $\Phi=-G M / r$ is the Newtonian potential of the central mass $M, e$ is the specific internal energy, $\boldsymbol{\Pi}$ is the viscous stress tensor (we include all components), and $Q$ is the viscous dissipation function. Since we assume an ADAF, there is no radiative cooling. We adopt the ideal gas equation of state, $P=(\gamma-1) \rho e$, where $\gamma$ is the adiabatic index, and write the kinematic shear viscosity coefficient as $\nu=\alpha c_{s}^{2} / \Omega_{K}$, where $c_{s}=(P / \rho)^{1 / 2}$ is the isothermal sound speed and $\Omega_{K}=\left(G M / r^{3}\right)^{1 / 2}$ is the Keplerian angular velocity. Equations (2.1)-(2.3) are independent of the black hole mass $M$ when the radius $r$ is scaled by the gravitational radius $r_{g}=2 G M / c^{2}$ and time is scaled by $r_{g} / c$.

Each time step of the numerical computation is split into two sub-steps: hydrodynamical and viscous. The hydrodynamical sub-step is calculated by using the PPM algorithm (Colella \& Woodward 1984), and the viscous sub-step is solved by using a standard explicit algorithm for the viscous contribution to the equation of motion (2.2) and the energy equation (2.3).

We solve equations (2.1)-(2.3) on a 3D Cartesian grid consisting of a sequence of six nested sub-grids. Each sub-grid $k$ is $26 \times 26 \times 52$ in size and is uniform, extending over the range $\left(0, R_{k}^{\text {out }}\right),\left(0, R_{k}^{\text {out }}\right),\left(-R_{k}^{\text {out }}, R_{k}^{\text {out }}\right)$ in $x, y, z$, respectively. The innermost grid has $R_{1}^{\text {out }}=26 r_{g}$ and

each successive $R_{k}^{\text {out }}$ is equal to $2 R_{k-1}^{\text {out }}$, such that the outermost sub-grid extends to $R_{6}^{\text {out }}=832 r_{g}$. Figure 1 illustrates the geometry of the grid. Due to numerical limitations we compute the flow only over a quarter of the full cubic volume and apply azimuthal periodic boundary conditions in the $(x, z)$ and $(y, z)$ planes. At the outer boundary $R_{6}^{\text {out }}$ we use a free outflow boundary condition, and in the innermost sub-grid we assume that matter drains freely inside a sphere of radius $r \simeq 3 r_{g}$. The inner boundary condition mimics the effect of the last stable orbit around a Schwarzschild black hole. 
The time-step $\Delta t$ for the numerical integration is chosen in accordance with the Courant condition for the hydrodynamical sub-step. For this $\Delta t$, the viscous integration is numerically stable because we choose sufficiently low values for the viscosity parameter $\alpha$. We assume that mass is injected into the computational domain over an equatorial slender torus with radius $r_{i n j} \approx 800 r_{g}$. The matter is injected at a fixed rate $\dot{M}_{i n j}$ and has rotational velocity (with respect to the $z$-axis) equal to 0.95 times the local Keplerian velocity. As a result of viscous interactions, part of the injected matter moves inwards and forms an accretion flow, while the rest leaves the computational domain through the outer boundary. We follow the time evolution of the flow on time scales longer than the viscous time scale at $r_{i n j}$. This allows the flow to attain steady state in a time-averaged sense. Note that when $\rho$ is scaled by $\dot{M}_{i n j}$, the results are independent of the value of $\dot{M}_{i n j}$. Therefore, the simulations depend on only two dimensionless parameters: $\alpha, \gamma$.

\section{Results}

We have calculated four models (A, B, C, D) which differ in the choice of $\alpha$ and $\gamma$ (see Table 1). All the models show complicated time-dependent convective motions, analogous to those described in detail by Igumenshchev et al. (1996), Igumenshchev \& Abramowicz (1999, 2000) and Stone et al. (1999). The amplitude of the variability is not large, and the average properties of the flow are well represented by time-averaging. To good accuracy, the time-averaged flow is axisymmetric in all the models.

Figure 2 shows the time-averaged radial profiles in the equatorial plane $(z=0)$ of $\rho, \Omega$ and $c_{s}$ for the four models. In the inner regions, $r \lesssim 10^{2} r_{g}$, the profiles are well approximated by power-laws: $\Omega \propto r^{-3 / 2}, c_{s} \propto r^{-1 / 2}, \rho \propto r^{-\beta}$, where the index $\beta$ is $\approx 0.5$ for Models $\mathrm{A}$ and $\mathrm{C}$ and $\approx 0.8$ for Models B and D. (For $r>2 \times 10^{2} r_{g}$, the flows are strongly affected by the outer boundary at $r \approx 8 \times 10^{2} r_{g}$ and are not power-law in character.) The scalings of $\Omega$ and $c_{s}$ and that of $\rho$ in Models $\mathrm{A}$ and $\mathrm{C}$ are identical to those predicted by the analytical work of NIA and QG. The $\beta \approx 0.8$ scaling of Models $\mathrm{B}$ and $\mathrm{D}$ is a little surprising, and suggests that $\beta$ depends on the value of $\gamma$. A similar dependence of $\beta$ on $\gamma$ was found also in the axisymmetric 2D simulations of Igumenshchev \& Abramowicz (2000).

Figure 3 shows a snapshot of the flow in the equatorial plane for Model C. The other three models are qualitatively similar. The most important feature is that the density and velocity fluctuations are almost axisymmetric, as seen by the fact that the eddies are significantly elongated in the azimuthal direction. This suggests that the present 3D results are not likely to be very different from those obtained with $2 \mathrm{D}$ axisymmetric simulations. The minimum length-scale of perturbations in the radial $r$ and polar $\theta$ directions depends on the viscosity parameter $\alpha$, being larger for larger values of $\alpha$.

The left panel in Fig. 4 shows a snapshot meridional cross-section of Model $\mathrm{C}$ in the $(y, z)$-plane. The shading shows the distribution of the vorticity vector, $\vec{\omega}=\operatorname{rot} \vec{v}$. Regions with 
a positive $x$-component of the vorticity $\omega_{x}$ are shown in grey, and regions with negative $\omega_{x}$ are

shown in white. It is seen that the flow is dominated by radial streams of inflowing and outflowing material which are sandwiched in the $\theta$-direction. The streams are chaotic and move around so that they are washed out when a time average is taken. Models A and B are very similar to Model $\mathrm{C}$, except that they have $2-3$ inflowing streams at a given moment, whereas Model $\mathrm{C}$ has usually $5-6$ streams.

Model D is qualitatively different from the other three models, as shown by the right panel in Fig. 4. What is plotted here is the time-averaged flow pattern and vorticity. We see that the model has quasi-stationary structures which survive even after averaging over about 100 periods of the Keplerian rotation at $r=100 r_{g}$. The flow pattern is almost symmetrical with respect to the equatorial plane, and shows the presence of a stable and regular arrangement of circulation cells in the meridional plane.

The direction of transport of angular momentum is given by the sign of the $(r \varphi)$-component of the Reynolds stress tensor, $\left\langle v_{r}^{\prime} v_{\varphi}^{\prime}\right\rangle$. NIA showed that this quantity is negative in $2 \mathrm{D}$ simulations of convective ADAFs, but noted that it was a consequence of the axisymmetry of the simulations and that real 3D convection might behave differently. In Figure 5 we show the radial dependence of the Reynolds stress averaged over $\theta$ for the four 3D models described in this paper. We see that, except for a small boundary region, the stress is negative over the bulk of the flow in all the models. This confirms that the $2 \mathrm{D}$ results published in previous papers are physically meaningful. Figure 5 shows that the magnitude of the Reynolds stress is positively correlated with the values of both $\alpha$ and $\gamma$. Model $\mathrm{D}$ has a surprisingly weak stress. This may be connected to the quasi-stationary meridional circulation seen in this model.

\section{Discussion and Conclusions}

We have used numerical simulations to study three-dimensional hydrodynamic accretion flows with inefficient radiative cooling (ADAFs). The simulated flows have nearly Keplerian angular momentum on the outside, relatively weak viscosity, $\alpha=0.01,0.03$, and an adiabatic index $\gamma$ equal to either $5 / 3$ or $4 / 3$. We find that the convective motions are nearly axisymmetric (Fig. $3)$. As a result, the basic properties of the flows are very similar to those found in previous $2 \mathrm{D}$ axisymmetric simulations (Igumenshchev et al. 1996; Igumenshchev \& Abramowicz 1999, 2000; Stone et al. 1999; NIA).

Our most important result is that convection transports angular momentum inward (Fig. 5). This property of convection was identified by Ryu \& Goodman (1992) in analytical work on thin accretion disks. Recently, NIA showed that convection in 2D axisymmetric simulations of ADAFs also moves angular momentum inward. They and QG argued, however, on the basis of previous work by Stone \& Balbus (1996), that this result was a consequence of enforcing axisymmetry on the accreting gas. The present work shows that even in $3 \mathrm{D}$, where the flow is not constrained to be 
axisymmetric, convective motions do transport angular momentum inward. In fact, we find that $2 \mathrm{D}$ and $3 \mathrm{D}$ simulations show good quantitative agreement in the estimate of the $(r \varphi)$-component of the Reynolds stress tensor, and in the radial dependences of various fluid variables.

In the models, the turbulent pressure due to convection, $P_{\text {turb }}=\left\langle\rho v^{\prime 2}\right\rangle / 3$, where $v^{\prime}$ is the velocity fluctuation, is $\lesssim 10^{-3}$ of the gas pressure $P$. Therefore, convection does not have a direct dynamical effect on the flow. However, as argued by NIA and QG, convection can have a very strong indirect effect on the structure of the flow if it causes inward transport of angular momentum. The accretion flow can then achieve a critically balanced state in which the convective transport almost completely cancels the outward transport of angular momentum by viscosity. This leads to a flat radial density profile $\rho \propto r^{-1 / 2}$. Since the amount of transport possible with convection is limited, the viscosity parameter $\alpha$ must be less than a critical value $\alpha_{\text {crit }} \lesssim 0.1$ for the new structure to be realized (NIA). The value of $\alpha$ in an ADAF is not known at present. Model-fitting to the observed light curve and spectra of soft X-ray transients suggests that $\alpha \sim 0.2-0.3$ (Esin, McClintock \& Narayan 1997). Numerical MHD simulations in 3D should provide an independent estimate of $\alpha$ (assuming that viscosity in ADAFs is produced by magnetic stresses). If such computations confirm that $\alpha$ in ADAFs is large, then convection may turn out to have only limited dynamical effect on these flows. Conversely, if $\alpha<\alpha_{\text {crit }}$, one may expect ADAFs to resemble the analytical solutions described by NIA and QG.

Our results may be relevant also to the physics of rapidly rotating convective stars. In such stars convection may reduce or even suppress the outward transport of angular momentum by magnetic stresses (see Bisnovatyi-Kogan et al. 1979).

An important property of convective accretion flows is the outward transport of energy by convection. We find that $2 \mathrm{D}$ and $3 \mathrm{D}$ simulations give similar results; the effective outward luminosity due to convection is $\sim 10^{-3}-10^{-2} \dot{M} c^{2}$ for the values of $\alpha$ and $\gamma$ considered here, where $\dot{M}$ is the net mass accretion rate of the black hole. Very likely the energy will be radiated on the outside of the accretion flow. If so, the results have important implications for the spectra and luminosities of accreting black holes and neutron stars (Igumenshchev \& Abramowicz 2000). Convection could also introduce variability on different time-scales (Igumenshchev \& Abramowicz 1999).

The simulated flows presented in this paper show some interesting dependence on the adiabatic index $\gamma$. Models with $\gamma=4 / 3$ have a steeper density profile (Fig. 2) than models with $\gamma=5 / 3$. In addition, Model D $(\alpha=0.01, \gamma=4 / 3)$ shows a quasi-stationary meridional circulation (Fig. 4), whereas other models have chaotic convective motions. The reason for these differences is unclear.

This study was supported by a grant from the Royal Swedish Academy of Sciences and by grants PHY 9507695 and AST 9820686 from the National Science Foundation. 


\section{REFERENCES}

Bisnovatyi-Kogan, G. S., Blinnikov, S. I., Kostyuk, N. D., \& Fedorova, A. V. 1979, Soviet Astr., 23,432

Colella, P., \& Woodward, P. R. 1984, J. Comput. Phys., 54, 174

Esin, A. A., McClintock, J. E., \& Narayan, R. 1997, ApJ, 489, 865

Igumenshchev, I.V., Chen, X., \& Abramowicz, M.A. 1996, MNRAS, 278, 236

Igumenshchev, I.V., \& Abramowicz, M.A. 1999, MNRAS, 303, 309

Igumenshchev, I.V., \& Abramowicz, M.A. 2000, ApJ, submitted (astro-ph/0003397)

Narayan, R., \& Yi, I. 1994, ApJ, 428, L13

Narayan, R., \& Yi, I. 1995, ApJ, 444, 231

Narayan, R., Igumenshchev, I.V., \& Abramowicz, M.A. 2000, ApJ, in press (astro-ph/9912449, NIA)

Quataert, E. \& Gruzinov, A. 2000, ApJ, in press (astro-ph/9912440, QG)

Ryu, D., \& Goodman, J. 1992, ApJ, 388, 438

Stone, J.M., Pringle, J.E., \& Begelman, M.C. 1999, MNRAS, 310, 1002

Stone, J.M., \& Balbus, S. A. 1996, ApJ, 464, 364 
Table 1. Parameters of the models.

\begin{tabular}{lcc}
\hline \hline Model & $\alpha$ & $\gamma$ \\
\hline $\mathrm{A}$ & 0.03 & $5 / 3$ \\
$\mathrm{~B}$ & 0.03 & $4 / 3$ \\
$\mathrm{C}$ & 0.01 & $5 / 3$ \\
$\mathrm{D}$ & 0.01 & $4 / 3$ \\
\hline
\end{tabular}




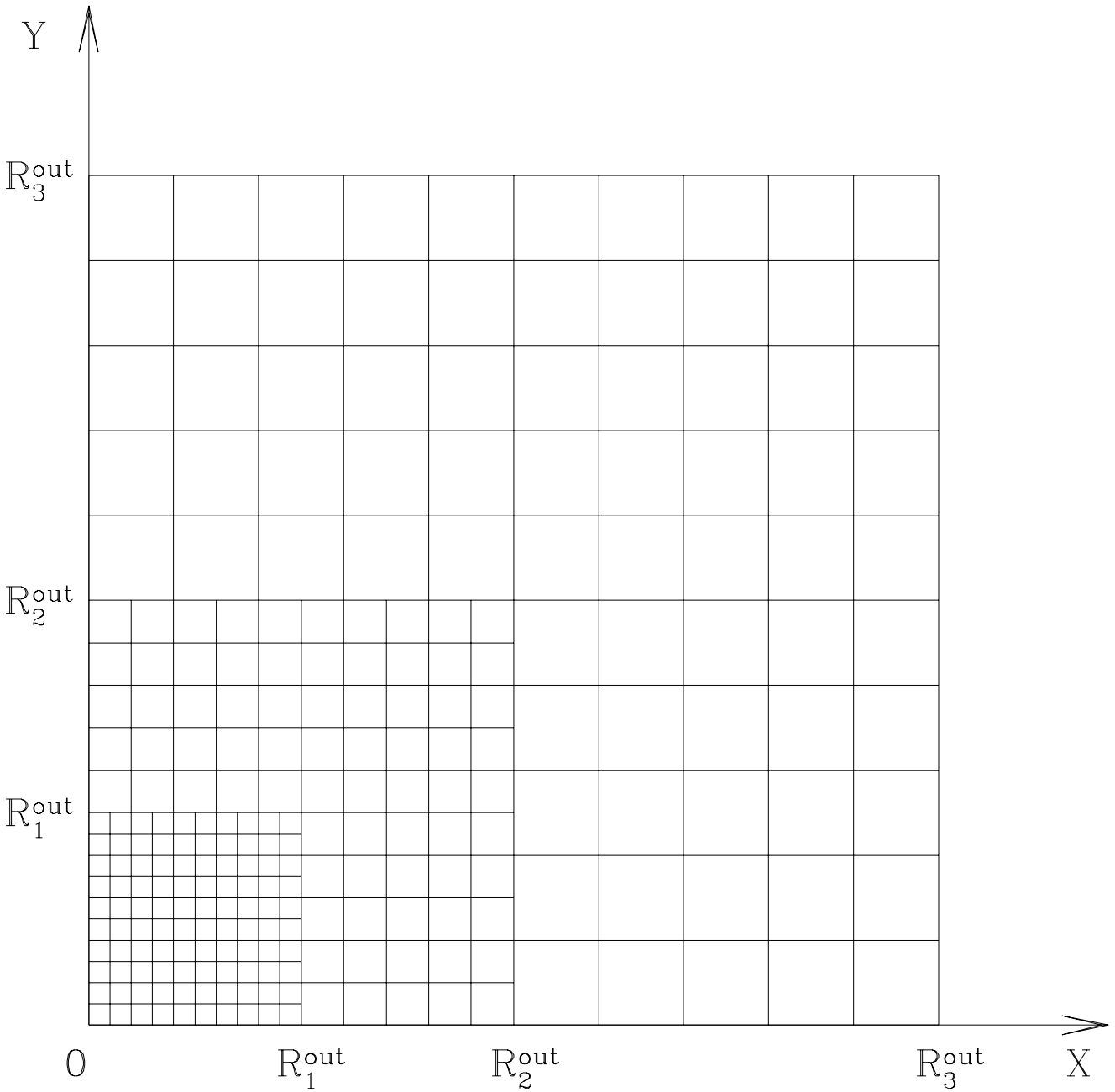

Fig. 1.- A 2D illustration of the nested Cartesian grid used in the calculations. The example shown has three sub-grids, each of size $10 \times 10$. 

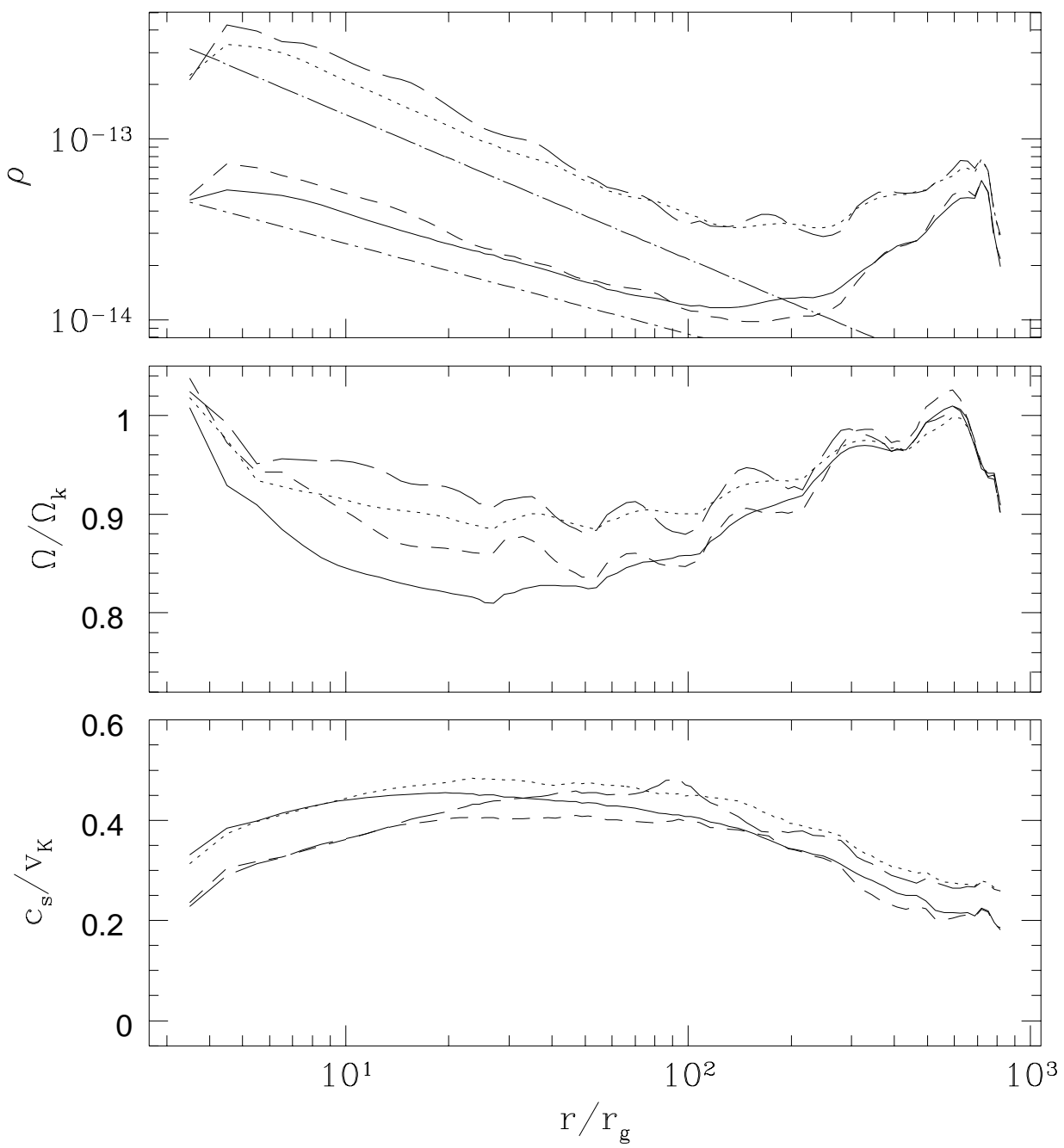

Fig. 2.- Radial profiles of the equatorial density $\rho$, angular velocity $\Omega_{/} \Omega_{K}$ and isothermal sound speed $c_{s} / r \Omega_{K}$ for Models A (solid lines), B (dotted lines), C (dashed lines) and D (long-dashed lines). The dot-dashed and dot-long-dashed lines in the top panel show power-law profiles, $r^{-0.5}$ and $r^{-0.8}$, for comparison. 

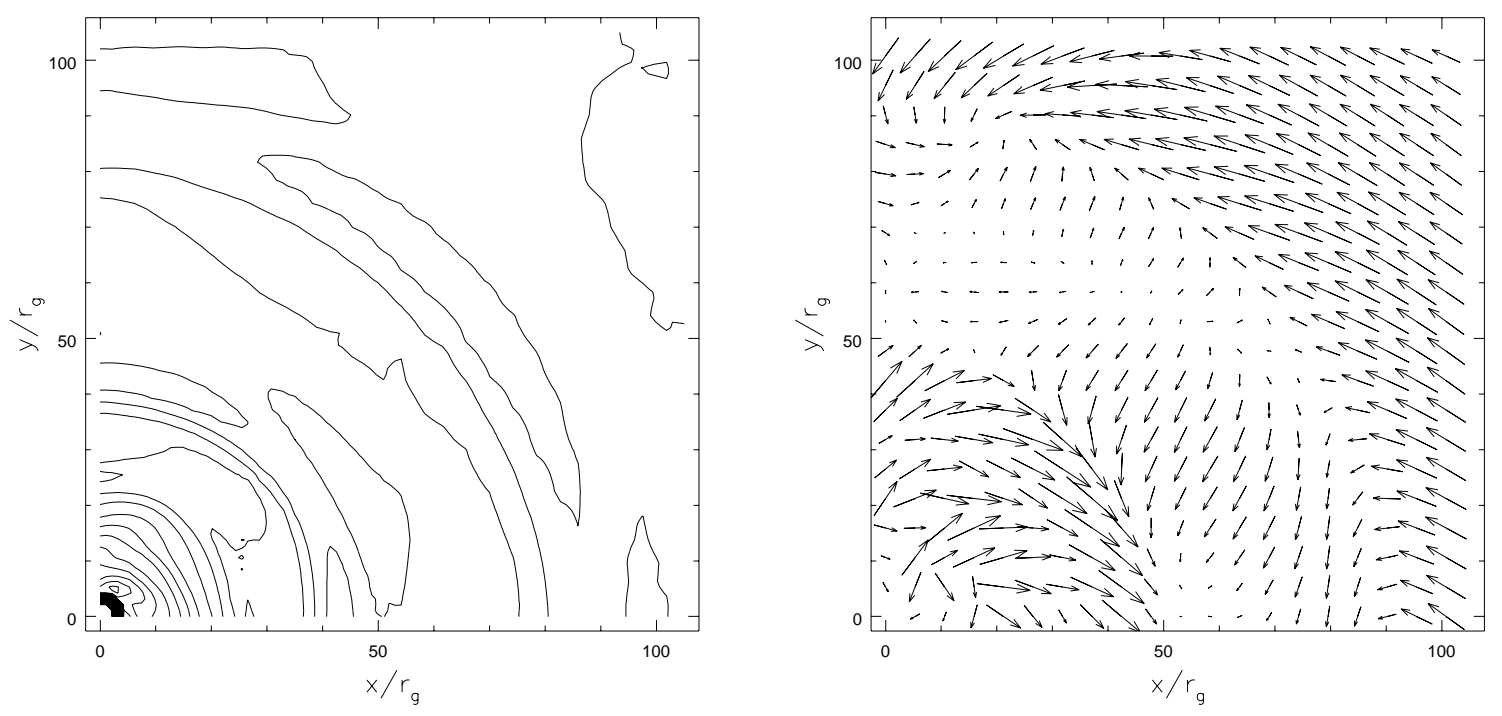

Fig. 3.- Snapshot view of the equatorial density (left) and velocity field (right) in Model C. The density contours are spaced with $\Delta \log \rho=0.05$. Arrows in the right panel show the dimensionless relative velocity $(\vec{v}-\langle\vec{v}\rangle) /|\langle\vec{v}\rangle|$, where $\langle\vec{v}\rangle$ is the time-averaged velocity. The background rotation of the accretion flow is anti-clockwise. Therefore, material rotating clockwise/anti-clockwise in the figure has less/more specific angular momentum than the background. 

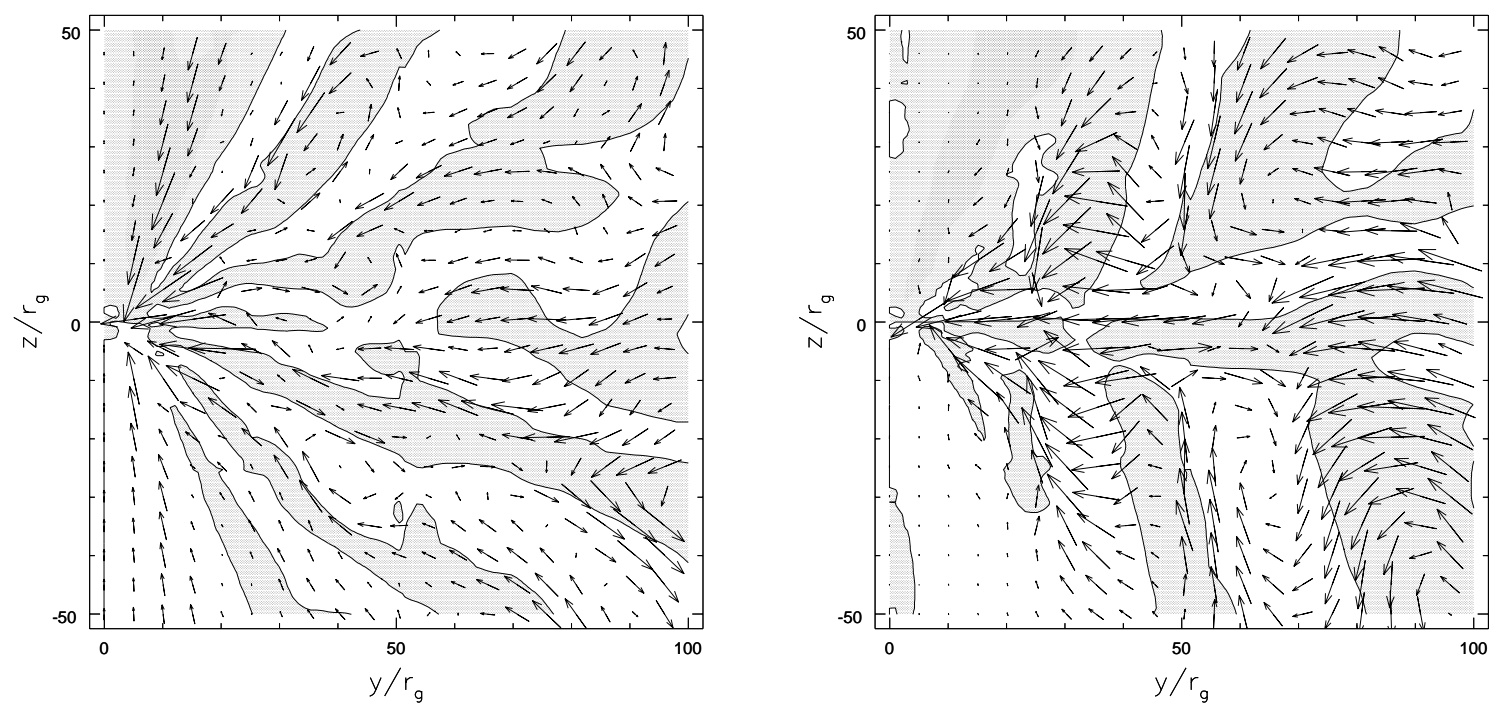

Fig. 4.- Vorticity and momentum vectors in the meridional $(y, z)$-plane corresponding to a snapshot of Model C (left) and a time-average of Model D (right). Arrows correspond to $r \rho \vec{v}$. 


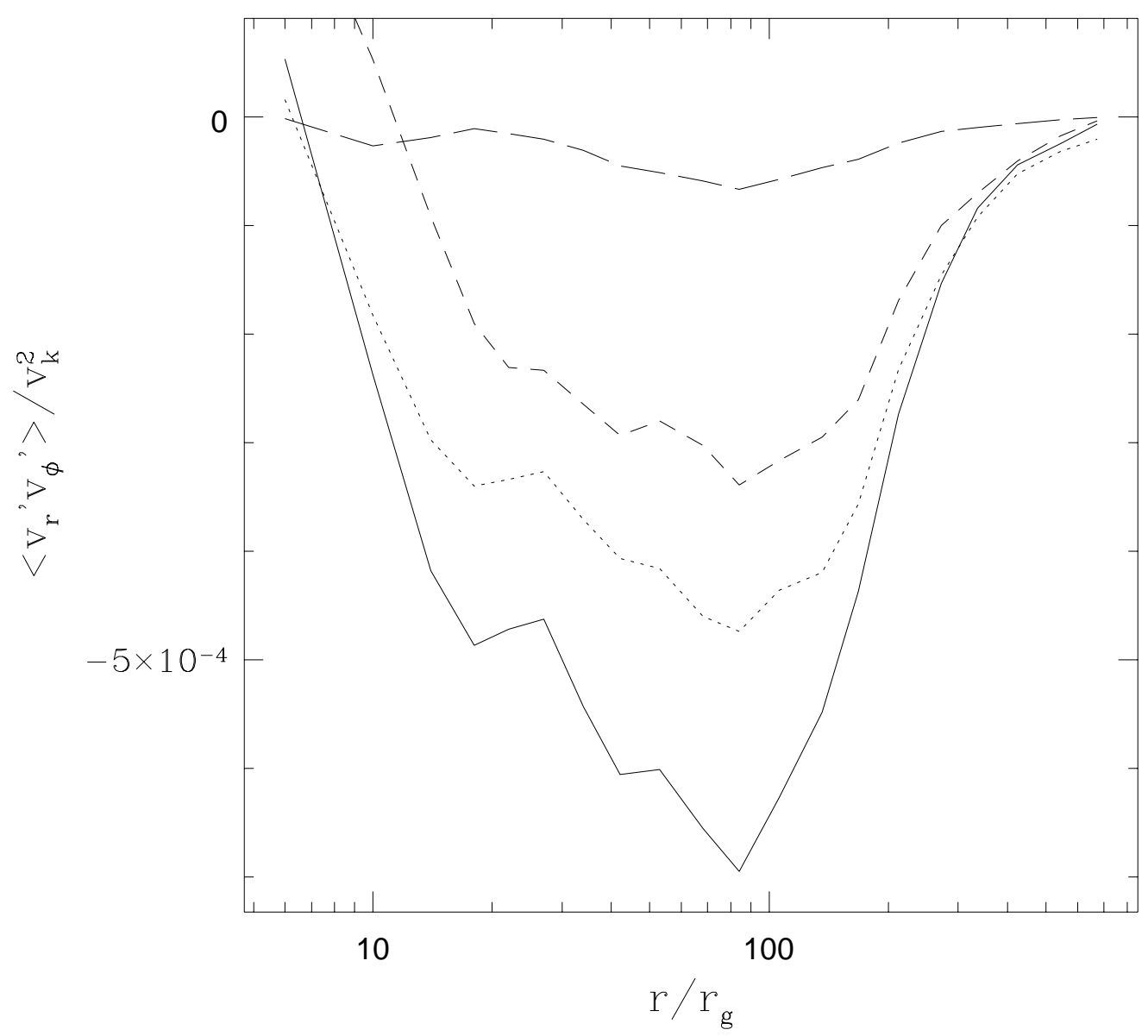

Fig. 5. - Radial profiles of the $(r \varphi)$-component of the Reynolds stress tensor $\left\langle v_{r}^{\prime} v_{\varphi}^{\prime}\right\rangle$ for Models A (solid line), B (dotted line), C (dashed line) and D (long-dashed line). The stresses have been averaged over polar angle $\theta$ and normalized to the square of the Keplerian velocity $v_{K}^{2}$. 